\title{
Repeated Aspergillus isolation in respiratory samples from non-immunocompromised patients not selected based on clinical diagnoses: colonisation or infection?
}

Jose Barberan ${ }^{*}$, Bernardino Alcazar ${ }^{2}$, Eduardo Malmierca ${ }^{3}$, Francisco Garcia de la Llana ${ }^{4}$, Jordi Dorca ${ }^{5}$, Daniel del Castillo ${ }^{6}$, Victoria Villena ${ }^{7}$, Melissa Hernandez-Febles ${ }^{8}$, Francisco-Javier Garcia-Perez ${ }^{9}$, Juan-Jose Granizo ${ }^{10}$, Maria-Jose Gimenez ${ }^{11}$ and Lorenzo Aguilar ${ }^{11}$ on behalf of the ASP Investigator Group

\begin{abstract}
Background: Isolation of Aspergillus from lower respiratory samples is associated with colonisation in high percentage of cases, making it of unclear significance. This study explored factors associated with diagnosis (infection vs. colonisation), treatment (administration or not of antifungals) and prognosis (mortality) in non-transplant/non-neutropenic patients showing repeated isolation of Aspergillus from lower respiratory samples.

Methods: Records of adult patients (29 Spanish hospitals) presenting $\geq 2$ respiratory cultures yielding Aspergillus were retrospectively reviewed and categorised as proven (histopathological confirmation) or probable aspergillosis (new respiratory signs/symptoms with suggestive chest imaging) or colonisation (symptoms not attributable to Aspergillus without dyspnoea exacerbation, bronchospasm or new infiltrates). Logistic regression models (step-wise) were performed using Aspergillosis (probable + proven), antifungal treatment and mortality as dependent variables. Significant $(p<0.001)$ models showing the highest $R^{2}$ were considered.

Results: A total of 245 patients were identified, 139 (56.7\%) with Aspergillosis. Aspergillosis was associated $\left(R^{2}=0.291\right)$ with ICU admission ( $\left.O R=2.82\right)$, congestive heart failure $(\mathrm{OR}=2.39)$ and steroids pre-admission $(\mathrm{OR}=2.19)$ as well as with cavitations in $\mathrm{X}$-ray/CT scan $(\mathrm{OR}=10.68)$, radiological worsening $(\mathrm{OR}=5.22)$ and $\mathrm{COPD}$ exacerbations/need for $\mathrm{O}_{2}$ interaction $(\mathrm{OR}=3.52)$. Antifungals were administered to $79.1 \%$ patients with Aspergillosis (100\% proven, $76.8 \%$ probable) and 29.2\% colonised, with 69.5\% patients receiving voriconazole alone or in combination. In colonised patients, administration of antifungals was associated with ICU admission at hospitalisation $(\mathrm{OR}=12.38)$. In Aspergillosis patients its administration was positively associated $\left(R^{2}=0.312\right)$ with bronchospasm $(\mathrm{OR}=9.21)$ and days in ICU $(\mathrm{OR}=1.82)$ and negatively with Gold III + IV $(\mathrm{OR}=0.26)$, stroke $(\mathrm{OR}=0.024)$ and quinolone treatment $(\mathrm{OR}=0.29)$. Mortality was $78.6 \%$ in proven, $41.6 \%$ in probable and $12.3 \%$ in colonised patients, and was positively associated in Aspergillosis patients $\left(R^{2}=0.290\right)$ with radiological worsening $(\mathrm{OR}=3.04)$, APACHE-II $(\mathrm{OR}=1.09)$ and number of antibiotics for treatment $(\mathrm{OR}=1.51)$ and negatively with species other than $A$. fumigatus $(\mathrm{OR}=0.14)$ and aspergillar tracheobronchitis $(\mathrm{OR}=0.27)$.

(Continued on next page)
\end{abstract}

\footnotetext{
* Correspondence: josebarberan@teleline.es

'Infectious Diseases Department, Hospital Central de la Defensa Gómez Ulla, Gta. del Ejército s/n, 28047 Madrid, Spain

Full list of author information is available at the end of the article
} 
(Continued from previous page)

Conclusions: Administration of antifungals was not always closely linked to the diagnostic categorisation (colonisation vs. Aspergillosis), being negatively associated with severe COPD (GOLD III + IV) and concomitant treatment with quinolones in patients with Aspergillosis, probably due to the similarity of signs/symptoms between this entity and pulmonary bacterial infections.

Keywords: Aspergillus, COPD, Clinical management, Aspergillosis

\section{Background}

Isolation of Aspergillus from lower respiratory samples is associated with colonisation in high percentage of cases, being of unclear significance because it may represent a temporary passage, a long-term benign carriage or a sign preceding invasive disease since the incubation period is unknown [1,2]. Invasive disease has been described as disease of immunocompromised patients, and for high risk patients (those with bone marrow or solid organ transplant, neutropenia or haematological cancer [3]) standard definitions of opportunistic infection (proven, probable or possible) to express disease certainty have been internationally agreed [4]. In these type of patients, isolation of Aspergillus from lower respiratory tract samples is potentially significant [5], and acquires relevance since early diagnosis seems crucial to improve prognosis of this potentially treatable disease $[1,6]$.

Invasive aspergillosis is increasingly being recognised as an emerging opportunistic infection in non-neutropenic patients, with reports on patients receiving immunossupressive therapy that do not impair neutrophils count, patients with cancer (receiving or not treatment), connective diseases requiring corticosteroids, liver cirrhosis, elderly patients (that comprise a growing segment of hospitalised patients), patients with less immunodeficiency such as patients with chronic obstructive pulmonary disease (COPD), specially those under steroid therapy, and ICU patients without apparent predisposing immunodeficiency [7-12].

Sensitivity of culture isolation for invasive infection varies from $5 \%$ to $75 \%$, depending on the population [10]. Since culture has also poor specificity, it has been suggested the need for repeated isolation of the same Aspergillus species as part of the diagnosis of invasive aspergillosis [13], in a field where the use of diagnostic tools widely differs between different hospitals.

The aim of this study was to explore implications for the patient's management of the repeated isolation of Aspergillus from lower respiratory samples in nontransplant, non-neutropenic patients not selected based on clinical diagnoses, by means of identification of factors associated with diagnosis (infection vs. colonisation), with treatment (administration of antifungals) and with prognosis (mortality).

\section{Patients and methods}

Clinical records of adult patients presenting at least two cultures of evaluable lower respiratory tract samples yielding Aspergillus in 29 Spanish hospitals were retrospectively reviewed to include up to 10 patients in each centre (the 10 most recent consecutive evaluable patients with complete clinical records in the period 2001-2010). Eligible clinical records were identified through microbiology records. Transplant recipients and patients presenting neutropenia $(<1000$ neutrophils/ $\mathrm{mm}^{3}$ ), diagnosis of aspergilloma or allergic bronchopulmonary aspergillosis were excluded. The study protocol was approved by the Ethics Committee of Hospital Central de la Defensa Gomez Ulla, Madrid, Spain.

Demographic data, underlying illnesses, clinical and radiological data, laboratory data, previous corticosteroids intake (within 3 months prior to admission), previous antibiotic and antifungal treatments (within 30 days prior to admission), antifungal treatment during hospitalization and outcome were recorded. The age-unadjusted Charlson comorbidity index [14] (age was considered in separate), the modified McCabe score (Sabadell score) [15], the functional classification according to the New York Heart Association (NYHA) [16] and the Acute Physiologic and Chronic Health Evaluation (APACHE) II score were calculated with recorded data, as well as the Global Initiative for Chronic Obstructive Lung Disease (GOLD) classification for COPD patients [17]. A diagnostic category was assigned to each patient using criteria adapted from those defined by Bulpa et al. for COPD patients [1], not considering the "possible" category since in the present study all patients required at least two positive Aspergillus cultures as inclusion criteria. Patients were classified as with proven aspergillosis (histopathological confirmation), probable aspergillosis (presence of new respiratory signs/ symptoms with suggestive chest imaging) or colonisation (symptoms not attributable to Aspergillus without dyspnoea exacerbation, bronchospasm or new pulmonary infiltrates). For data analysis a category named "Aspergillosis" was considered by pooling patients with probable aspergillosis and proven aspergillosis. Diagnoses of tracheobronchitis were always based on bronchoscopy reports included in clinical records. Simple tracheobronchitis was considered when the bronchoscopy report described mucosal inflammation and mucus secretions 
and invasive tracheobronchitis when ulceration and pseudomembrane formation was observed. Chronic pulmonary aspergillosis was considered when long-term fibrotic lesions with or without necrosis or cavitations had been recorded in the patient's clinical records.

Comparisons between proportions were performed by the $x^{2}$ test and the Fisher's exact test, when necessary. For quantitative variables, since data did not showed normality in the Kolmogorov - Smirnoff test, the Kruskal-Wallis and Mann-Whitney tests, when necessary, were used. Bivariate analyses were performed comparing all variables between colonised vs. patients with Aspergillosis, between antifungal treated vs. non-treated patients and between patients who died vs. those that did not. Different logistic regression models (step-wise procedure) were performed using as dependent variables i) Aspergillosis, ii) antifungal treatment and iii) mortality, and as independent variables those showing differences $(\mathrm{p} \leq 0.1)$ in bivariate analyses. Interactions and linear dependence between independent variables were previously controlled. Statistical analyses were performed using SPSS v 14 programme (SPSS Inc, Chicago, IL). The models showing the highest $\mathrm{R}^{2}$ were considered.

\section{Results}

A total of 245 clinical records were included in the study, corresponding to patients attended in the study centres from October 2002 to July 2010 (19 centres included 10 patients each and 10 centres included the remaining 55 patients). Of the 245 patients considered, 106 were categorised as colonised and 139 as patients with Aspergillosis (125 as probable and 14 as proven aspergillosis). Six patients (2.4\%) had cystic fibrosis (5 patients classified as colonized and 1 as with probable aspergillosis). Aspergillus fumigatus was the most frequent species isolated, accounting for $66.9 \%$ of total isolates, followed by Aspergillus niger in 6.1\% patients and Aspergillus flavus in $3.7 \%$ patients. In a total of $23.3 \%$ patients cultures had been informed as Aspergillus spp. or other species. A. fumigatus was isolated in all cases categorised as proven aspergillosis. The serum galactomannan level had been determined in 51 (20.8\%) patients. The result was positive $(\geq 1 \mathrm{ng} / \mathrm{ml})$ in $33.3 \%$ (14 out of 42) patients with Aspergillosis while it was negative in all patients categorised as colonised in which the test was performed $(n=9)$. Histopathological examination was performed in 19 patients, the 14 patients classified as with proven aspergillosis and another 5 patients where biopsies were performed and were negative (patients classified as with probable aspergillosis). In the 14 patients with aspergillosis, histopathological confirmation was obtained by fine needle aspiration biopsy (2 patients), pulmonary biopsy (8 patients), renal biopsy (1 patient) and necropsy (3 patients).

\section{Descriptive analysis}

Table 1 shows epidemiological data and comorbidities present in the study patients distributed by diagnostic category. Mean age of patients was $68.7 \pm 15.2$ years (with $42.9 \%$ patients $\geq 75$ years of age and $22.0 \%$ $\geq 80$ years), without differences between categories. The percentage of males was significantly higher among patients with Aspergillosis (74.8\% vs. $63.2 \%$ in colonised; $\mathrm{p}=0.05)$. Significantly higher percentage of patients with severe COPD (GOLD III + IV) $(p=0.037)$, congestive heart failure $(p=0.031)$ and malignancies $(p=0.011)$ were found among patients with Aspergillosis, without

Table 1 Epidemiological data and comorbidities of patients included in the study distributed by diagnostic category; [n (\%)] except where indicated

\begin{tabular}{|c|c|c|c|c|c|}
\hline & \multirow[t]{2}{*}{ Total } & \multirow[t]{2}{*}{ Colonised } & \multicolumn{3}{|c|}{ Aspergillosis } \\
\hline & & & Probable & Proven & Probable + Proven \\
\hline No. Patients & 245 & 106 & 125 & 14 & 139 \\
\hline Age (mean $\pm S D)$ & $68.7 \pm 15.2$ & $69.1 \pm 16.1$ & $68.9 \pm 14.6$ & $64.1 \pm 14.5$ & $68.4 \pm 14.6$ \\
\hline Males & $171(69.8)$ & $67(63.2)$ & $94(75.2)$ & $10(71.4)$ & $104(74.8)^{\mathrm{a}}$ \\
\hline \multicolumn{6}{|l|}{ Comorbidities $^{\mathbf{b}}$} \\
\hline COPD & $173(70.6)$ & $73(68.9)$ & $91(72.8)$ & $9(64.3)$ & $100(71.9)$ \\
\hline Gold III + IV & $111(67.3)$ & $40(57.2)$ & $66(76.7)^{\mathrm{a}}$ & $5(55.6)$ & $71(74.7)^{\mathrm{a}}$ \\
\hline Diabetes Mellitus II & $43(17.6)$ & 19 (17.9) & $23(18.4)$ & $1(7.1)$ & $24(17.3)$ \\
\hline Congestive heart failure & $45(18.4)$ & $13(12.3)$ & $27(21.6)$ & $5(35.7)^{\mathrm{a}}$ & $32(23.0)^{\mathrm{a}}$ \\
\hline Malignancies & $38(15.5)$ & $10(9.4)$ & $27(21.6)^{a}$ & $1(7.1)$ & $28(20.1)^{a}$ \\
\hline Renal impairment & $24(9.8)$ & $10(9.4)$ & $12(9.6)$ & $2(14.3)$ & $14(10.1)$ \\
\hline Charlson (mean \pm SD) & $2.61 \pm 2.16$ & $2.42 \pm 2.03$ & $2.81 \pm 2.29$ & $2.21 \pm 1.76$ & $2.75 \pm 2.75$ \\
\hline APACHE $($ mean \pm SD) & $13.16 \pm 6.86$ & $11.63 \pm 6.12$ & $14.13 \pm 6.67^{\mathrm{a}}$ & $16.14 \pm 10.90$ & $14.33 \pm 7.18^{\mathrm{a}}$ \\
\hline ICU admission at any time during hospital stay & $58(23.7)$ & $15(14.2)$ & $36(28.8)^{a}$ & $7(50.0)^{\mathrm{a}}$ & $43(30.9)^{\mathrm{a}}$ \\
\hline
\end{tabular}


significant differences in the Charlson index between categories. The mean APACHE II score was significantly higher in patients with Aspergillosis (14.33 vs. 11.63 in colonised; $p=0.004)$, reaching a value of 16.14 in patients with proven aspergillosis. Overall prognosis (McCabe score) was non fatal in $60.0 \%$ patients, longterm fatal ( $>6$ months) in $31.0 \%$ patients and short-term fatal ( $<6$ months) in $8.9 \%$ patients, without differences between categories.

Only 18 patients (7.3\%) were ambulatory, $72.2 \%$ categorised as colonised and $27.8 \%$ as with probable aspergillosis. A total of $48.2 \%$ patients had been initially admitted in Pneumology, 23.3\% in Internal Medicine, $11.4 \%$ in the Intensive Care Unit (ICU) and 9.8\% in other wards, without differences between diagnostic categories. Admission in the ICU as initial ward was more frequent among proven cases $(28.6 \%)$ than among probable $(11.2 \%)$ or colonised cases $(9.4 \%)$, although differences did not reach statistical significance $(\mathrm{p}=0.085$ vs. probable and $\mathrm{p}=0.058$ vs. colonised). ICU admission at any time during hospitalisation (Table 1) was significantly higher among patients with Aspergillosis (30.9\%) than among those colonised $(14.2 \%)(\mathrm{p}=0.001)$, with $50.0 \%$ admission rate among those with proven aspergillosis ( $p=0.004$ vs. colonised) and $28.8 \%$ among those with probable aspergillosis ( $\mathrm{p}=0.007$ vs. colonised).

Table 2 shows imaging data of patients distributed by diagnostic categories. Significantly $(\mathrm{p}<0.001)$ higher percentage of patients showing infiltrates and cavitations was found (both in X-ray and CT scan) in the group of patients with Aspergillosis than in colonised patients. Worsening of radiological findings in successive X-ray was also significantly more frequent among patients with Aspergillosis (36.7\% vs. 6.7\% colonised, $\mathrm{p}<0.001)$. CT scan was more frequently requested in patients further categorised as having Aspergillosis than in those categorised as colonised $(64.7 \%$ vs. $46.2 \%, \mathrm{p}=0.004)$, being up to $78.6 \%$ among patients with proven aspergillosis. The halo and the air crescent signs were only found in 4 and 3 cases, respectively, of Aspergillosis.

Table 3 shows intake of steroids, antibiotics and antifungals dividing patients by diagnostic categories. Prior to admission, the percentage of patients with Aspergillosis that had received corticosteroids was significantly higher than those colonised $(51.8 \%$ vs. $27.4 \%, \mathrm{p}<0.001)$ due to the subgroup of patients receiving accumulated doses $>100 \mathrm{mg}(81.9 \%$ vs. $58.6 \%, \mathrm{p}=0.033)$. During hospitalisation, $75.5 \%$ patients received steroids without differences between diagnostic categories. A total of 35.9\% patients received antibiotics prior to admission and $82.0 \%$ received them during hospitalisation, without differences between diagnostic categories in both cases. Antifungal treatment was initiated after culture request in $79.1 \%$ patients further categorised as having Aspergillosis (100\% proven cases and $76.8 \%$ probable cases) and in $29.2 \%$ colonised patients $(\mathrm{p}<0.001)$. Voriconazole was the most frequent antifungal used, with up to $69.5 \%$ patients receiving this compound alone or in combination with other antifungals. In patients with Aspergillosis, antifungal treatment was voriconazole as monotherapy in $51.8 \%$ cases, voriconazole plus other antifungals in $22.7 \%$ cases and other antifungals (amphotericin, caspofungin, fluconazole and itraconazole) alone or in combination in $25.5 \%$ cases.

Table 4 shows clinical presentations and outcome by diagnostic category. A total of $54.6 \%$ patients with Aspergillosis presented invasive pulmonary aspergillosis, $18.4 \%$ simple tracheobronchitis, $16.3 \%$ chronic pulmonary

Table 2 Imaging data corresponding to patients included in the study distributed by diagnostic category; [n (\%)]

\begin{tabular}{|c|c|c|c|c|c|}
\hline & \multirow[t]{2}{*}{ Total } & \multirow[t]{2}{*}{ Colonised } & \multicolumn{3}{|c|}{ Aspergillosis } \\
\hline & & & Probable & Proven & Probable + Proven \\
\hline No. patients with chest $\mathrm{Rx}$ & 244 & 105 & 125 & 14 & 139 \\
\hline Infiltrates & $134(54.9)$ & $46(43.8)$ & $76(60.8)^{a}$ & $12(85.7)^{a}$ & $88(63.3)^{a}$ \\
\hline Nodules & $37(15.2)$ & $13(12.4)$ & $24(19.2)$ & $0(0.0)$ & $24(17.3)$ \\
\hline Cavitations & $21(8.6)$ & $0(0.0)$ & $19(15.2)^{\mathrm{a}}$ & $2(14.3)^{\mathrm{a}}$ & $21(15.1)^{\mathrm{a}}$ \\
\hline Pleural effusion & $25(10.2)$ & $7(6.7)$ & $16(12.8)$ & $2(14.3)$ & $18(12.9)$ \\
\hline Worsening radiological findings & $58(23.8)$ & $7(6.7)$ & $43(34.4)^{a}$ & $8(57.1)^{a}$ & $51(36.7)^{a}$ \\
\hline No. patients with CT Scan & $139(56.7)$ & $49(46.2)$ & $79(63.2)^{\mathrm{a}}$ & $11(78.6)^{a}$ & $90(64.7)^{\mathrm{a}}$ \\
\hline Infiltrates & $78(56.1)$ & 19 (39.6) & $48(60.0)^{a}$ & $11(100)^{\mathrm{a}}$ & $59(64.8)^{a}$ \\
\hline Nodules & $56(40.3)$ & $14(28.6)$ & $39(49.4)^{a}$ & $3(27.3)$ & $42(46.7)^{a}$ \\
\hline Cavitations & $33(23.7)$ & $5(10.2)$ & $23(29.1)^{a}$ & $5(45.5)^{a}$ & $28(31.1)^{a}$ \\
\hline Halo sign & $4(2.9)$ & $0(0.0)$ & $3(3.8)$ & $1(9.1)$ & $4(4.4)$ \\
\hline Air crescent sign & $3(2.2)$ & $0(0.0)$ & $3(3.8)$ & $0(0.0)$ & $3(3.3)$ \\
\hline
\end{tabular}

$a_{p} \leq 0.05$ vs. colonised. 
Table 3 Steroids, antibiotic and antifungals intake [n (\%)] by diagnostic category

\begin{tabular}{|c|c|c|c|c|c|}
\hline & \multirow[t]{2}{*}{ Total } & \multirow[t]{2}{*}{ Colonised } & \multicolumn{3}{|c|}{ Aspergillosis } \\
\hline & & & Probable & Proven & Probable + Proven \\
\hline No. patients & 245 & 106 & 125 & 14 & 139 \\
\hline Steroids intake before admission & $101(41.2)$ & $29(27.4)$ & $64(51.2)^{a}$ & $8(57.1)^{a}$ & $72(51.8)^{\mathrm{a}}$ \\
\hline$\geq 20 \mathrm{mg} /$ day & $59(58.4)$ & $15(51.7)$ & $40(62.5)$ & $4(50.0)$ & $44(61.1)$ \\
\hline Accumulated dose $>100 \mathrm{mg}$ & $76(75.2)$ & $17(58.6)$ & $52(81.3)^{\mathrm{a}}$ & $7(87.5)$ & $59(81.9)^{\mathrm{a}}$ \\
\hline Accumulated dose $>700 \mathrm{mg}$ & $20(19.8)$ & $6(20.7)$ & $11(17.2)$ & $3(37.5)$ & $14(19.4)$ \\
\hline Antibiotic treatment before admission & $88(35.9)$ & $34(32.1)$ & 47 (37.6) & $7(50.0)$ & $54(38.8)$ \\
\hline Antifungal treatment after culture request & $141(57.6)$ & $31(29.2)$ & $96(76.8)^{\mathrm{a}}$ & $14(100)^{a}$ & $110(79.1)^{\mathrm{a}}$ \\
\hline Voriconazole as monotherapy & $68(48.2)$ & $11(35.5)$ & $55(57.3)$ & $2(14.3)$ & $57(51.8)$ \\
\hline Voriconazole + other antifungals & $30(21.3)$ & $5(16.1)$ & $18(18.8)$ & $7(50.0)$ & $25(22.7)$ \\
\hline Total patients receiving voriconazole & $98(69.5)$ & $16(51.6)$ & $73(76.1)$ & $9(64.3)$ & $82(74.5)$ \\
\hline Other antifungals & $43(30.5)$ & $15(48.4)$ & $23(24.0)$ & $5(35.7)$ & $28(25.5)$ \\
\hline
\end{tabular}

aspergillosis and 8.5\% invasive tracheobronchitis. All 14 patients with proven aspergillosis presented pulmonary infection: $71.4 \%$ invasive aspergillosis and $28.6 \%$ chronic aspergillosis. Significantly higher mortality was found in proven $(78.6 \%)$ than in probable aspergillosis $(41.6 \%)(\mathrm{p}=$ $0.018)$ or colonisation $(12.3 \%)(\mathrm{p}<0.001)$, as well as in probable aspergillosis vs. colonisation $(\mathrm{p}<0.001)$. Aspergillus was involved as cause of death (with or without others) in $78.5 \%$ patients with proven aspergillosis compared with $32.0 \%$ in those with probable aspergillosis $(\mathrm{p}=0.002)$ and $0 \%$ in those colonised $(\mathrm{p}<0.001)$.

Factors defining infection vs. colonisation

In the multivariate analysis exploring variables associated with diagnostic categories (colonisation vs. Aspergillosis), which included all variables showing significance $(\mathrm{p}<0.1)$ in the bivariate analysis previously performed, the logistic regression was statistically significant ( $\mathrm{p}<0.001, \mathrm{R}^{2}$ Cox $=0.291$ ). Aspergillosis was associated with ICU admission at any time $(\mathrm{p}=0.012$; $\mathrm{OR}=2.82$, 95\% $\mathrm{CI}=1.26-6.37$ ), congestive heart failure $(\mathrm{p}=0.044 ; \mathrm{OR}=2.39,95 \% \mathrm{CI}=1.03-5.55)$ and the intake of steroids prior to admission ( $\mathrm{p}=0.021$; OR $=2.19,95 \%$ $\mathrm{CI}=1.13-4.27)$ in addition to variables suggesting Aspergillosis as the presence of cavitations in X-ray and/or CT scan $(\mathrm{p}<0.001 ; \mathrm{OR}=10.68,95 \% \mathrm{CI}=3.61-31.64)$, the worsening of radiological findings $(\mathrm{p}=0.001$; $\mathrm{OR}=5.22$, 95\% CI $=2.04-13.33$ ) and the interaction between COPD exacerbations and need for $\mathrm{O}_{2}(\mathrm{p}<0.001 ; \mathrm{OR}=3.52$, $95 \% \mathrm{CI}=1.75-7.11)$.

Variables associated with administration of antifungals Table 5 shows variables with statistical significance $(\mathrm{p}<0.1)$ in the bivariate analysis performed comparing patients treated with antifungals vs. those not treated, considering the total population, colonised patients

Table 4 Clinical presentations and outcome by diagnostic category

\begin{tabular}{|c|c|c|c|c|c|}
\hline & \multirow[t]{2}{*}{ Total } & \multirow[t]{2}{*}{ Colonised } & \multicolumn{3}{|c|}{ Aspergillosis } \\
\hline & & & Probable & Proven & Probable + Proven \\
\hline No. patients & 245 & 106 & 125 & 14 & 139 \\
\hline Invasive pulmonary aspergillosis & $77(31.4)$ & & $68(54.4)$ & $10(71.4)$ & $78(56.1)$ \\
\hline Chronic pulmonary aspergillosis & $23(9.4)$ & & 19 (15.2) & $4(28.6)$ & $23(16.5)$ \\
\hline Simple tracheobronchitis & $26(10.6)$ & & $26(20.8)$ & $0(0.0)$ & $26(18.7)$ \\
\hline Invasive tracheobronchitis & $12(4.9)$ & & $12(9.4)$ & $0(0.0)$ & $12(8.6)$ \\
\hline Mortality & $76(31.0)$ & $13(12.3)$ & $52(41.6)^{a}$ & $11(78.6)^{a, b}$ & $63(45.3)^{a}$ \\
\hline a) Death attributable to Aspergillus & $7(2.9)$ & $0(0.0)$ & $4(3.2)$ & $3(21.4)^{a, b}$ & $7(5.0)$ \\
\hline b) Death attributable to Aspergillus and to other causes & $44(18.0)$ & $0(0.0)$ & $36(28.8)^{a}$ & $8(57.1)^{a}$ & $44(31.7)^{a}$ \\
\hline a) + b) & $51(20.9)$ & $0(0.0)$ & $40(32.0)^{\mathrm{a}}$ & $11(78.5)^{a, b}$ & $51(36.7)$ \\
\hline Death not attributable to Aspergillus & $25(10.2)$ & $13(12.3)$ & $12(9.6)$ & $0(0.0)$ & $12(8.6)$ \\
\hline
\end{tabular}

Results are expressed as " $\mathrm{n}(\%)$ "

${ }^{a} p<0.001$ vs. colonised; ${ }^{b} p<0.02$ vs. probable. 
Table 5 Significant variables in the bivariate analysis of patients receiving vs. not receiving antifungals, [n (\%)] except where indicated

\begin{tabular}{|c|c|c|c|c|c|c|c|c|c|}
\hline & \multicolumn{3}{|c|}{ Total patients $(n=245)$} & \multicolumn{3}{|c|}{ Colonised $(n=106)$} & \multicolumn{3}{|c|}{ Aspergillosis $(n=139)$} \\
\hline & $\begin{array}{l}\text { Treated } \\
(n=104)\end{array}$ & $\begin{array}{c}\text { Non-treated } \\
(n=141)\end{array}$ & $p$ & $\begin{array}{l}\text { Treated } \\
(n=31)\end{array}$ & $\begin{array}{l}\text { Non-treated } \\
\quad(n=75)\end{array}$ & $p$ & $\begin{array}{l}\text { Treated } \\
(n=110)\end{array}$ & $\begin{array}{l}\text { Non-treated } \\
\quad(n=29)\end{array}$ & $p$ \\
\hline Age $(X \pm S D)$ & $66.7 \pm 15.9$ & $71.4 \pm 13.8$ & 0.016 & $66.0 \pm 16.7$ & $70.4 \pm 15.7$ & 0.086 & $66.9 \pm 15.8$ & $73.9 \pm 6.6$ & 0.001 \\
\hline Days to isolation $(X \pm S D)$ & $12.3 \pm 12.2$ & $7.6 \pm 7.6$ & 0.001 & $8.9 \pm 6.6$ & $7.0 \pm 6.5$ & 0.096 & $13.2 \pm 13.1$ & $9.1 \pm 9.6$ & 0.046 \\
\hline COPD & $90(63.8)$ & $83(79.8)$ & 0.007 & $15(48.4)$ & $58(77.3)$ & 0.005 & $75(68.2)$ & $25(86.2)$ & 0.064 \\
\hline GOLD III + IV & $57(40.4)$ & $54(51.9)$ & 0.074 & $8(53.4)$ & $32(58.2)$ & 0.568 & $49(65.3)$ & $22(88.0)$ & 0.041 \\
\hline COPD exacerbation & $22(15.6)$ & $3(2.9)$ & 0.625 & $6(19.4)$ & $29(38.7)$ & 0.070 & $56(50.9)$ & $20(69.0)$ & 0.082 \\
\hline Severe liver disease & $3(2.1)$ & $8(7.7)$ & 0.058 & $1(3.2)$ & $6(8.0)$ & 0.671 & $2(1.8)$ & $2(6.9)$ & 0.192 \\
\hline Stroke & $4(2.8)$ & $6(5.8)$ & 0.332 & $1(3.2)$ & $2(2.7)$ & 1.000 & $3(2.7)$ & $4(13.8)$ & 0.035 \\
\hline APACHE II $(X \pm S D)$ & $13.8 \pm 7.6$ & $12.3 \pm 5.6$ & 0.076 & $12.6 \pm 7.5$ & $11.2 \pm 5.4$ & 0.291 & $14.1 \pm 7.6$ & $15.1 \pm 5.1$ & 0.439 \\
\hline ICU admission & $47(33.3)$ & $11(10.6)$ & 0.001 & $10(32.3)$ & $5(6.7)$ & 0.001 & 37 (33.6) & $6(20.7)$ & 0.259 \\
\hline ICU stay (days; $\mathrm{X} \pm \mathrm{SD}$ ) & $7.6 \pm 7.9$ & $10.0 \pm 3.4$ & 0.113 & $2.1 \pm 4.8$ & $0.3 \pm 1.8$ & 0.014 & $7.9 \pm 8.3$ & $2.0 \pm 2.8$ & 0.046 \\
\hline Pleuritic pain & 19 (13.5) & $6(5.8)$ & 0.049 & $2(6.5)$ & $5(6.7)$ & 1.00 & $17(15.5)$ & $1(3.4)$ & 0.121 \\
\hline Bronchospasm & $66(46.8)$ & $23(22.1)$ & 0.001 & $8(25.8)$ & $17(22.7)$ & 0.803 & $58(52.7)$ & $6(20.7)$ & 0.003 \\
\hline Infiltrates (X-ray / CT) & $102(72.3)$ & $47(45.2)$ & 0.001 & $18(58.1)$ & $31(41.3)$ & 0.137 & $84(76.4)$ & $16(55.2)$ & 0.024 \\
\hline Cavitations (X-ray / CT) & $31(22.0)$ & $7(6.7)$ & 0.001 & $1(3.2)$ & $4(5.3)$ & 0.642 & $30(27.3)$ & $3(10.3)$ & 0.084 \\
\hline Nodules (X-ray / CT) & $48(34.0)$ & $22(21.2)$ & 0.027 & $5(16.1)$ & $16(21.3)$ & 0.541 & $43(39.1)$ & $6(20.7)$ & 0.081 \\
\hline Treatment with Quinolones & $41(29.1)$ & $42(40.4)$ & 0.065 & $12(38.7)$ & $27(36.0)$ & 0.827 & $29(26.4)$ & $15(51.7)$ & 0.009 \\
\hline
\end{tabular}

and patients with Aspergillosis. The multivariate analysis performed including the variables shown in the Table arose the following results:

a) Total population

The multivariate analysis was statistically significant $\left(\mathrm{p}<0.001, \mathrm{R}^{2} \mathrm{Cox}=0.221\right)$ and antifungal treatment was associated with stay in the ICU $(\mathrm{p}<0.001$; OR $=4.78,95 \% \mathrm{CI}=2.20$ 10.36) and the presence of two or more radiological findings (infiltrates and/or nodules and/or cavitations) in X-ray and/or CT scan $(\mathrm{p}<0.001 ; \mathrm{OR}=2.43,95 \% \mathrm{CI}=1.67-3.53)$ and negatively associated with severe liver disease $(\mathrm{p}=0.015 ; \mathrm{OR}=0.15,95 \% \mathrm{CI}=0.03-0.69)$.

b) Patients categorised as colonised In the multivariate analysis the logistic regression was significant $(\mathrm{p}=0.001)$ but showed a weak association $\left(\mathrm{R}^{2}\right.$ Cox $\left.=0.118\right)$. Antifungal treatment was only associated with ICU admission at hospitalization ( $\mathrm{p}=0.002$; $\mathrm{OR}=12.38,95 \% \mathrm{CI}=2.44-62.92$ ).

c) Patients categorised as with Aspergillosis The multivariate analysis was statistically significant $\left(\mathrm{p}<0.001, \mathrm{R}^{2}\right.$ Cox $\left.=0.312\right)$ and antifungal treatment was positively associated with days of stay in the ICU ( $\mathrm{p}=0.021$; OR $=1.82,95 \%$ $\mathrm{CI}=1.09-3.05)$ and presence of bronchospasm $(\mathrm{p}=0.001 ; \mathrm{OR}=9.21,95 \% \mathrm{CI}=2.63-32.21)$ and negatively associated with GOLD III + IV $(\mathrm{p}=0.024 ; \mathrm{OR}=0.26,95 \% \mathrm{CI}=0.08-0.84)$, stroke $(\mathrm{p}=0.009 ; \mathrm{OR}=0.024,95 \% \mathrm{CI}=0.01-0.40)$ and concomitant treatment with quinolones $(\mathrm{p}=0.034$; $\mathrm{OR}=0.29,95 \% \mathrm{CI}=0.09-0.91$ ).

Variables associated with mortality

Table 6 includes variables showing statistical significance in the bivariate analysis comparing dead patients vs. those that survived considering the total population, colonised patients and patients with Aspergillosis. The multivariate analysis performed including the variables shown in the Table arose the following results:

a) Total population

The multivariate analysis was statistically significant $\left(\mathrm{p}<0.001, \mathrm{R}^{2}\right.$ Cox $\left.=0.261\right)$ and mortality was associated with malignancies ( $\mathrm{p}<0.001 ; \mathrm{OR}=4.65,95 \% \mathrm{CI}=2.05-10.58)$, intake of steroids (whether prior or during admission) ( $\mathrm{p}=0.005 ; \mathrm{OR}=4.33,95 \% \mathrm{CI}=1.55-12.09)$, antifungal treatment $(\mathrm{p}<0.001 ; \mathrm{OR}=3.68,95 \%$ $\mathrm{CI}=1.81-7.49$ ), and the APACHE II score $(\mathrm{p}<0.001 ; \mathrm{OR}=1.14,95 \% \mathrm{CI}=1.07-1.20)$ and negatively associated with COPD $(\mathrm{p}=0.045$; $\mathrm{OR}=0.44,95 \% \mathrm{CI}=0.20-0.98$ ).

b) Patients categorised as colonised In the multivariate analysis the logistic regression 
Table 6 Significant clinical variables in the bivariate analysis of patients with respect to mortality, [n (\%)] except where indicated

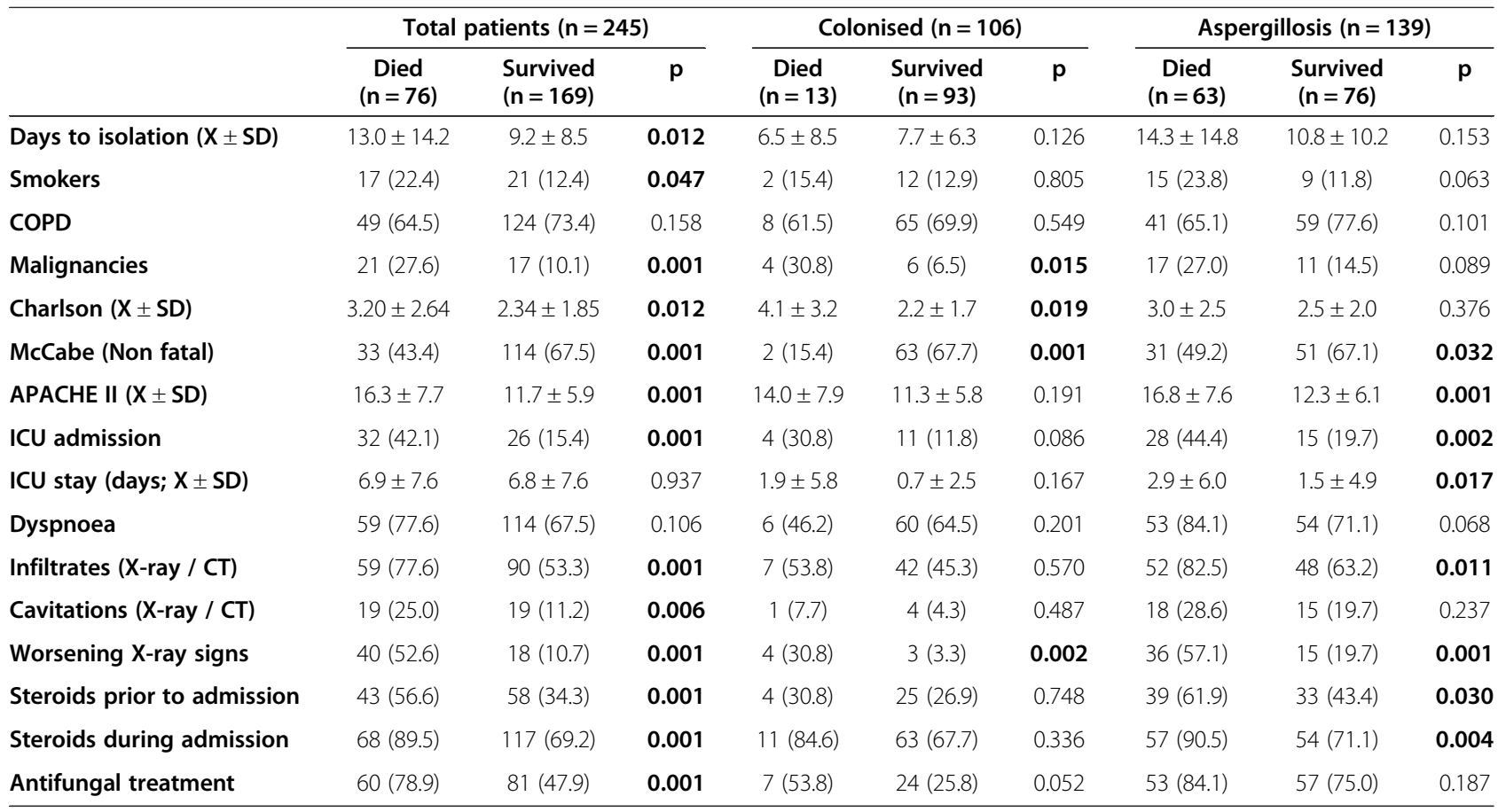

was significant $\left(\mathrm{p}=0.001 ; \mathrm{R}^{2} \operatorname{Cox}=0.246\right)$. Mortality was associated with presence of malignancies ( $\mathrm{p}=0.001 ; \mathrm{OR}=31.87,95 \% \mathrm{CI}=3.78-258.59)$ and worsening of $\mathrm{X}$-ray signs $(\mathrm{p}=0.002 ; \mathrm{OR}=25.38$, $95 \% \mathrm{CI}=3.13-205.89$ ), and it was negatively associated with COPD ( $\mathrm{p}=0.030$; OR $=0.11$, 95\% CI $=0.02-0.81$.

c) Patients categorised as with Aspergillosis The multivariate analysis was statistically significant $\left(\mathrm{p}<0.001, \mathrm{R}^{2}\right.$ Cox $\left.=0.290\right)$ and mortality was positively associated with worsening of $\mathrm{X}$-ray signs $(\mathrm{p}=0.011$; OR $=3.04$, $95 \% \mathrm{CI}=1.28-7.21)$, number of antibiotics postculture $(\mathrm{p}=0.013$; OR $=1.51,95 \% \mathrm{CI}=1.09-2.08)$, APACHE II score $(\mathrm{p}=0.011 ; \mathrm{OR}=1.09$, $95 \% \mathrm{CI}=1.02-1.17$ ), and negatively associated with diagnosis of aspergillar tracheobronchitis ( $\mathrm{p}=0.009 ; \mathrm{OR}=0.27,95 \% \mathrm{CI}=0.10-0.73$ ) and isolation of species other than $A$. fumigatus ( $\mathrm{p}=0.042 ; \mathrm{OR}=0.14,95 \% \mathrm{CI}=0.02-0.93$ ).

\section{Discussion}

Three questions arise when physicians face cultures positive to Aspergillus from lower respiratory samples in nonimmunocompromised/non-neutropenic patients: Is there colonisation or infection?, should the patient be treated with antifungals?, and which is the prognosis?, that is, how to interpret and manage patients from which Aspergillus is obtained. The retrospective analysis of this series of 245 immunocompetent patients showing at least two respiratory cultures positive to Aspergillus, that were further categorised as colonised, probable or proven aspergillosis, tried to explore how these three questions are addressed in the clinical practice. The diagnostic categorization of patients was based on criteria adapted from those defined by Bulpa et al. for COPD patients because we expected that most of the non-neutropenic, non-transplant patients in our series were this type of patients.

The answer to the first question is important since an early diagnosis is crucial to improve prognosis [1]. It has been postulated that isolation of an Aspergillus species from respiratory samples in critically ill patients (even when immunocompetent) should not be routinely discarded as colonisation [3], but in elderly patients (commonly having underlying diseases) isolation is usually interpreted as colonisation [10]. Confirmation of infection obliges the demonstration of histopathological evidence that is not usually feasible in this type of patients [18]. Early administration of antifungals may be life-saving, but overinterpretation of the potential clinical significance of Aspergillus isolation may drive to needless treatments, with their associated problems and costs $[5,18]$. How does clinicians solve the dilemma?

In this study 245 records from patients with at least two respiratory cultures positive to Aspergillus were identified, 139 of them (56.7\%) classified as with Aspergillosis 
(probable + proven) following adapted Bulpa et al. criteria. This contrasts with other published series where the percentage of patients showing Aspergillosis was markedly lower $[3,5,10]$, probably due to the at least two positive cultures required as inclusion criteria in our study. In this sense, a recent study in our country reported $22.1 \%$ of probable invasive pulmonary aspergillosis among hospitalized COPD patients with at least one positive culture to Aspergillus [19]. In addition to signs/symptoms suggestive of aspergillosis as cavitations in X-ray/CT scan, worsening of radiological findings and interaction of COPD and need for $\mathrm{O}_{2}$, according to the multivariate analysis performed, stay in the ICU, congestive heart failure and previous intake of steroids were also positively associated with Aspergillosis; all of them factors that had been previously described [1,6,7]. Although clinical signs/symptoms and radiological findings are usually non specific in critically ill patients [3], in our series requests of CT scan and the galactomannan test were significantly more frequent in patients further classified as with Aspergillosis than in those colonised, this suggesting a right clinical suspicion of colonisation/infection by treating physicians.

Initiation of antifungal treatment and time for initiation is a matter of considerable debate. Clinical manifestations of pulmonary aspergillosis may be initially indistinguishable from bacterial bronchopneumonia (fever, cough, purulent sputum) [20]. The recovery of the same Aspergillus species from several respiratory samples in the course of antibiotic-resistant pneumonia in patients with risk factors is clearly evocative of the diagnosis [21]. Therefore it has been proposed that the isolation of an Aspergillus species from the respiratory tract in critically ill patients with risk factors (COPD after corticosteroids exposure, severe underlying disease) and clinical features of pneumonia indicates prompt consideration of antifungal treatment [3,7].

In our series (with $72.6 \%$ of pulmonary aspergillosis among patients with Aspergillosis) antifungal treatment was not initiated in $23.2 \%$ patients with probable aspergillosis while it was administered to $29.2 \%$ of patients classified as colonised. Administration of antifungals was negatively associated with the presence of severe liver disease, probably because of the renal and hepatic toxicity of antifungals [11]. ICU admission and days in the ICU were associated with prescription of antifungals, both in colonised and aspergillosis patients. Interestingly, in patients with Aspergillosis antifungal treatment was negatively associated with Gold III + IV and treatment with quinolones. The negative association with treatment with wide-spectrum quinolones could probably be linked to the commented similarity of initial clinical manifestations of pulmonary aspergillosis with bacterial bronchopneumonia. In addition this could be the reason for the significantly lower number of severe
COPD patients found among treated vs. non-treated patients $(65.3 \%$ vs. $88.0 \%)$ in our series when analysing patients with Aspergillosis. However the percentage of COPD patients with Aspergillosis (Table 5) that received antifungal treatment in our series was $75 \%$ (75 out of $100)$, a percentage similar to the one $(71 \%)$ described in a published review of 65 cases [22].

Voriconazole was the most frequent antifungal drug, with $74.5 \%$ of treated patients with Aspergillosis receiving this drug alone or in combined regimens. For primary treatment of invasive aspergillosis, the triazole voriconazole is recommended for most patients [23] and, although its superiority over lipid formulations of Amphotericin B or echinocandins has not been proved in the subset of COPD patients [21], the superiority of voriconazole versus Amphotericin B has been demonstrated in invasive aspergillosis in immunocompromised patients [24].

The high mortality of invasive pulmonary aspergillosis in non-neutropenic critically ill patients has been attributed, at least partially, to difficulties in timely diagnosis caused by insensitive and non specific clinical signs and lack of unequivocal diagnostic criteria [11] precluding the early needed treatment. In the present study, as expected, significant higher mortality was found in proven than in probable aspergillosis and in the latter than among colonised patients $(78.6 \%$ vs. $41.6 \%$ vs. $12.3 \%)$. Interestingly, COPD was negatively associated with mortality in colonised patients but not in patients with Aspergillosis. Higher mortality rates (>90\%) in invasive pulmonary aspergillosis than those found in the present study have been reported in previously published series in COPD patients, but including only patients in the ICU [1,22]. Although the strength of the present study resides in the description of clinical features of aspergillosis in a large series of patients with at least two positive lower respiratory samples, several study limitations should be considered. The retrospective nature of the study based on information (clinical, radiological reports. .) recorded in clinical records from 29 different hospitals, implies bias derived from inter-hospital differences in patient's management. This could specially affect the management of patients concomitantly presenting other hospital infections and/or organ dysfunction. In addition most patients in the Aspergillosis category were classified as "probable aspergillosis", thus making difficult to establish firm conclusions due to the low number of proven cases or patients with $\mathrm{CT}$ scan images as halo sign or air crescent sign.

\section{Conclusions}

The analysis of the present series including 245 patients not selected based on clinical diagnoses but with at least two cultures of respiratory samples positive to Aspergillus showed that antifungal treatment is not always closely 
linked to the diagnostic categorisation (colonisation vs. Aspergillosis). Administration of antifungals in patients with Aspergillosis was negatively associated with severe COPD (GOLD III + IV) and concomitant treatment with quinolones, probably due to the similarity of signs/ symptoms between this entity and pulmonary bacterial infections.

\section{Abbreviations}

APACHE: Acute physiologic and chronic health evaluation; COPD: Chronic obstructive pulmonary disease; GOLD: Global initiative for chronic obstructive lung disease; ICU: Intensive care unit; NYHA: New York heart association; OR: Odds ratio.

\section{Competing interests}

J.B. and L.A. are members of Sociedad Española de Quimioterapia. J.B. and M-J. G. have received travel grants from Pfizer S.L.U. for presentation of study results in International congresses. Other authors: none to declare.

\section{Additional members of the ASP Investigator Group who contributed data}

I. Cuesta (Complejo Hospitalario de Jaén, Jaén), R. Sanchez-Silos (Complejo Hospitalario Universitario de Badajoz, Badajoz), R. Pascual and E. Lopez (H. Universitari de Bellvitge, l'Hospitalet de Llobregat), F. Perez-Grimaldi (H. de Jerez, Jerez de la Frontera), I. Campos (H. Universitario de Gran Canaria Dr. Negrín, Las Palmas de Gran Canaria), A. Pallares, A. Fernandez-Villar and I. Iglesias (H. Xeral de Vigo, Vigo), J. Blanquer, M.L. Briones and D. Navarro (H. Clínico Universitario de Valencia, Valencia), G. Fernandez-Calleja, J.R. Hernández, L. Martinez-Martinez (H. Universitario Marqués de Valdecilla, Santander), P. Llinares, M. Vares, D. Velasco (Complejo Hospitalario de A Coruña, A Coruña), M.A. Sepulveda and E. Heredero ( $H$. Virgen de la Salud, Toledo), S. Merlos (H. Universitario Virgen de las Nieves, Granada), A. Flor, I. Serra, R. Blavia and D. Estivill (H. Althaia Xarxa Assistencial, Manresa), E. Sanchez-Haya (H. de Donosti, San Sebastián), V. Acha and X. Beristain (H. Virgen del Camino, Pamplona), M. Chanza, T. Lloret and F. Sanz (H. General Universitario de Valencia, Valencia), C. del Valle and J.R. Maestre (H. Central de la Defensa Gomez Ulla, Madrid), M. Segarra (H. General de Elda, Elda), P. Serra (H. Universitari Germans Trias i Pujol, Badalona), R. Lama and M. Cause (H. General Universitario Reina Sofia, Cordoba), M. Riera, L. Martin-Pena, C. Marinescu and N. Borrell (H. Universitari Son Dureta, Palma de Mallorca), R. Malo and M. Valle (H. Universitario Puerta de Hierro, Majadahonda), E. Nuño and A. Infante (H. Universitario Virgen de la Victoria, Malaga), P. Hernandez (H. Universitario Nuestra Señora de Candelaria, Sta. Cruz de Tenerife), A. Ruedas, F. Hidalgo (H. Universitario Ramón y Cajal, Madrid), L. Vigil and A. Garcia-Garcia (Instituto Nacional de Silicosis, Oviedo), A. Robles ( $H$ Universitario La Paz, Madrid) and J. Prieto (Univ. Complutense, Madrid).

\section{Authors' contributions}

Conceived and designed the study: JB, J-JG, M-JG and LA. Collection of data: $J B, B A, E M, F G-L, J D, D d C, W, M H-F$ and F-JG-P. Analyzed the data: J-JG, M-JG and LA. Wrote the paper: JB, M-JG and LA. Made substantial intellectual contributions and approved the manuscript: all authors.

\section{Acknowledgements}

The authors are in debt with N. Mir and P. Calderón for their continuous support.

This study was supported by an unrestricted grant from the Spanish Society of Chemotherapy (Sociedad Española de Quimioterapia), Madrid, Spain that has received a Medical Education Grant from Pfizer S.L.U., Madrid, Spain. The sponsor had not role in study design, data collection analysis and interpretation, writing the report and in the decision to submit the article for publication.

Part of this study was presented at the 21st ECCMID/27th ICC, 7-10 May 2011, Milan (Italy).

\section{Author details}

'Infectious Diseases Department, Hospital Central de la Defensa Gómez Ulla, Gta. del Ejército s/n, 28047 Madrid, Spain. ${ }^{2}$ Pneumology Department, Complejo Hospitalario de Jaen, Jaen, Spain. ${ }^{3}$ Internal Medicine Department,
Hospital Infanta Sofia, San Sebastián de los Reyes, Madrid, Spain. ${ }^{4}$ Infectious Diseases Department, Complejo Hospitalario Universitario de Badajoz, Badajoz, Spain. ${ }^{5}$ Pneumology Department, Hospital Universitari de Bellvitge, Institut d'Investigació Biomèdica de Bellvitge (IDIBELL), Barcelona, Spain. ${ }^{6}$ Pneumology Department, Hospital de Jerez, Jerez de la Frontera, Cadiz, Spain. ${ }^{7}$ Pneumology Department, Hospital Universitario 12 de Octubre, Madrid, Spain. ${ }^{8}$ Microbiology Department, Hospital Universitario de Gran Canaria Dr. Negrin, La Palmas de Gran Canaria, Spain. ${ }^{9}$ Pneumology Department, Hospital Universitario de la Princesa, Madrid, Spain. ${ }^{10}$ Grana Datos, Pozuelo de Alarcón, Madrid, Spain. ${ }^{11}$ Microbiology Department, School of Medicine, Universidad Complutense, Madrid, Spain.

Received: 21 March 2012 Accepted: 7 November 2012 Published: 12 November 2012

\section{References}

1. Bulpa P, Dive A, Sibille Y: Invasive pulmonary aspergillosis in patients with chronic obstructive pulmonary disease. Eur Respir J 2007, 30:782-800.

2. Hope WW, Walsh TJ, Denning DW: The invasive and saprophytic syndromes due to Aspergillus spp. Med Mycol 2005, 43(Suppl 1):S207-S238.

3. Vandewoude KH, Blot SI, Depuydt P, Benoit D, Temmerman W, Colardyn F Vogelaers D: Clinical relevance of Aspergillus isolation from respiratory tract samples in critically ill patients. Crit Care 2006, 10:R31.

4. De Pauw B, Walsh TJ, Donnelly JP, Stevens DA, Edwards JE, Calandra T, Pappas PG, Maertens J, Lortholary O, Kauffman CA, Denning DW, Patterson TF, Maschmeyer G, Bille J, Dismukes WE, Herbrecht R, Hope WW, Kibbler CC, Kullberg BJ, Marr KA, Muñoz P, Odds FC, Perfect JR, Restrepo A, Ruhnke M, Segal BH, Sobel JD, Sorrell TC, Viscoli C, Wingard JR, et al: Revised definitions of invasive fungal disease from the European Organization for Research and Treatment of Cancer/Invasive Fungal Infections Cooperative Group and the National Institute of Allergy and Infectious Diseases Mycoses Study Group (EORTC/MSG) Consensus Group. Clin Infect Dis 2008, 46:1813-1821.

5. Bouza E, Guinea J, Peláez T, Pérez-Molina J, Alcalá L, Muñoz P: Workload due to Aspergillus fumigatus and significance of the organism in the microbiology laboratory of a general hospital. J Clin Microbiol 2005, 43:2075-2079

6. Garbino J, Fluckiger U, Elzi L, Imhof A, Bille J, Zimmerli S: Survey of aspergillosis in non-neutropenic patients in Swiss teaching hospitals. Clin Microbiol Infect 2011, 17:1366-1371

7. Garnacho-Montero J, Amaya-Villar R, Ortiz-Leyba C, León C, Alvarez-Lerma F, Nolla-Salas J, Iruretagoyena JR, Barcenilla F: Isolation of Aspergillus spp. from the respiratory tract in critically ill patients: risk factors, clinical presentation and outcome. Crit Care 2005, 9:R191-R199.

8. Kaiser P, Thurnheer R, Moll C, Frauchiger B, Rochat P, Krause M: Invasive aspergillosis in non-neutropenic patients. Eur J Intern Med 2009, 20:e131-e133

9. Khasawneh F, Mohamad T, Moughrabieh MK, Lai Z, Ager J, Soubani AO: Isolation of Aspergillus in critically ill patients: a potential marker of poor outcome. J Crit Care 2006, 21:322-327.

10. Soubani AO, Khanchandani G, Ahmed HP: Clinical significance of lower respiratory tract Aspergillus culture in elderly hospitalized patients. Eur J Clin Microbiol Infect Dis 2004, 23:491-494.

11. Trof RJ, Beishuizen A, Debets-Ossenkopp YJ, Girbes AR, Groeneveld AB Management of invasive pulmonary aspergillosis in non-neutropenic critically ill patients. Intensive Care Med 2007, 33:1694-1703.

12. Vandewoude $\mathrm{KH}$, Blot SI, Benoit D, Colardyn F, Vogelaers D: Invasive aspergillosis in critically ill patients: attributable mortality and excesses in length of ICU stay and ventilator dependence. J Hosp Infect 2004, 56:269-276.

13. Tashiro T, Izumikawa K, Tashiro M, Takazono T, Morinaga Y, Yamamoto $K$ Imamura Y, Miyazaki T, Seki M, Kakeya H, Yamamoto Y, Yanagihara K, Yasuoka A, Kohno S: Diagnostic significance of Aspergillus species isolated from respiratory samples in an adult pneumology ward. Med Mycol 2011, 49:581-587.

14. Charlson ME, Pompei P, Ales KL, Mackenzie CR: A new method of classifying prognostic comorbidity in longitudinal studies: development and validation. J Chronic Dis 1987, 40:373-383.

15. Fernandez R, Baigorri F, Navarro G, Artigas A: A modified McCabe score for stratification of patients after intensive care unit discharge: the Sabadell score. Crit Care 2006, 10(6):R179. 
16. New York Heart Association: New York Heart Association Functional Classification. http://my.americanheart.org/professional/ StatementsGuidelines/ByPublicationDate/PreviousYears/Classification-ofFunctional-Capacity-and-Objective-Assessment_UCM_423811_Article.jsp.

17. Pauwels RA, Buist AS, Calverley PM, Jenkins CR, Hurd SS, GOLD Scientific Committee: Global strategy for the diagnosis, management, and prevention of chronic obstructive pulmonary disease. NHLBI/WHO Global Initiative for Chronic Obstructive Lung Disease (GOLD) Workshop summary. Am J Respir Crit Care Med 2001, 163:1256-1276.

18. Garnacho-Montero J, Amaya-Villar R: A validated clinical approach for the management of aspergillosis in critically ill patients: ready, steady, go! Crit Care 2006, 10:132.

19. Guinea J, Torres-Narbona M, Gijón P, Muñoz P, Pozo F, Peláez T, de Miguel J, Bouza E: Pulmonary aspergillosis in patients with chronic obstructive pulmonary disease: incidence, risk factors, and outcome. Clin Microbiol Infect 2010, 16:870-877.

20. Olaechea Astigarraga PM, Alvarez Lerma F, Zaldíbar Enriquez E: Invasive pulmonary aspergillosis in the non-neutropenic critical patient: future challenges [Article in Spanish]. Med Intensiva 2006, 30:386-391.

21. Ader F: Invasive pulmonary aspergillosis in patients with chronic obstructive pulmonary disease: an emerging fungal disease. Curr Infect Dis Rep 2010, 12:409-416.

22. Samarakoon P, Soubani AO: Invasive pulmonary aspergillosis in patients with COPD: a report of five cases and systematic review of the literature. Chron Respir Dis 2008, 5:19-27.

23. Walsh TJ, Anaissie EJ, Denning DW, Herbrecht R, Kontoyiannis DP, Marr KA, Morrison VA, Segal BH, Steinbach WJ, Stevens DA, van Burik JA, Wingard JR, Patterson TF, Infectious Diseases Society of America: Treatment of aspergillosis: clinical practice guidelines of the Infectious Diseases Society of America. Clin Infect Dis 2008, 46:327-360.

24. Herbrecht R, Denning DW, Patterson TF, Bennett JE, Greene RE, Oestmann JW, Kern WW, Marr KA, Ribaud P, Lortholary O, Sylvester R, Rubin RH, Wingard JR, Stark P, Durand C, Caillot D, Thiel E, Chandrasekar PH, Hodges MR, Schlamm HT, Troke PF, de Pauw B, Invasive Fungal Infections Group of the European Organisation for Research and Treatment of Cancer and the Global Aspergillus Study Group: Voriconazole versus amphotericin B for primary therapy of invasive aspergillosis. N Engl J Med 2002, 347:408-415.

doi:10.1186/1471-2334-12-295

Cite this article as: Barberan et al:: Repeated Aspergillus isolation in respiratory samples from non-immunocompromised patients not selected based on clinical diagnoses: colonisation or infection? BMC Infectious Diseases 2012 12:295.

\section{Submit your next manuscript to BioMed Central and take full advantage of:}

- Convenient online submission

- Thorough peer review

- No space constraints or color figure charges

- Immediate publication on acceptance

- Inclusion in PubMed, CAS, Scopus and Google Scholar

- Research which is freely available for redistribution 